\title{
Introducing FDG PET/CT-guided chemoradiotherapy for stage III NSCLC in low- and middle-income countries: preliminary results from the IAEA PERTAIN trial
}

\author{
T. Konert ${ }^{1}$ (D) W. V. Vogel ${ }^{1,2} \cdot$ D. Paez ${ }^{3} \cdot$ A. Polo $^{3} \cdot$ E. Fidarova ${ }^{3} \cdot$ H. Carvalho ${ }^{4}$ - P. S. Duarte ${ }^{4} \cdot$ A. C. Zuliani $^{5} \cdot$ \\ A. O. Santos ${ }^{5}$ - D. Altuhhova ${ }^{6}$ - L. Karusoo ${ }^{6}$ - R. Kapoor ${ }^{7}$ - A. Sood ${ }^{7}$ - J. Khader ${ }^{8}$ - A. Al-lbraheem ${ }^{8} \cdot$ Y. Numair $^{9}$. \\ S. Abubaker ${ }^{9}$ - C. Soydal ${ }^{10}$ - T. Kütük ${ }^{10}$. T. A. Le ${ }^{11}$ - N. X. Canh ${ }^{11}$ • B. Q. Bieu ${ }^{12}$ • L. N. Ha ${ }^{12}$ • J. S. A. Belderbos ${ }^{2}$. \\ M. P. MacManus ${ }^{13,14}$. D. Thorwarth ${ }^{15}$. G. G. Hanna ${ }^{13,14}$
}

Received: 6 March 2019 / Accepted: 30 June 2019 / Published online: 31 July 2019

(C) The Author(s) 2019

\begin{abstract}
Purpose Patients with stage III non-small-cell lung cancer (NSCLC) treated with chemoradiotherapy (CRT) in low- and middleincome countries (LMIC) continue to have a poor prognosis. It is known that FDG PET/CT improves staging, treatment selection and target volume delineation (TVD), and although its use has grown rapidly, it is still not widely available in LMIC. CRT is often used as sequential treatment, but is known to be more effective when given concurrently. The aim of the PERTAIN study was to assess the impact of introducing FDG PET/CT-guided concurrent CRT, supported by training and quality control (QC), on the overall survival (OS) and progression-free survival (PFS) of patients with stage III NSCLC.

Methods The study included patients with stage III NSCLC from nine medical centres in seven countries. A retrospective cohort was managed according to local practices between January 2010 and July 2014, which involved only optional diagnostic FDG PET/CT for staging (not for TVD), followed by sequential or concurrent CRT. A prospective cohort between August 2015 and October 2018 was treated according to the study protocol including FDG PET/CT in treatment position for staging and multimodal TVD followed by concurrent CRT by specialists trained in protocol-specific TVD and with TVD QC. Kaplan-Meier analysis was used to assess OS and PFS in the retrospective and prospective cohorts.

Results Guidelines for FDG PET/CT image acquisition and TVD were developed and published. All specialists involved in the PERTAIN study received training between June 2014 and May 2016. The PET/CT scanners used received EARL accreditation. In November 2018 a planned interim analysis was performed including 230 patients in the retrospective cohort with a median follow-up of 14 months and 128 patients in the prospective cohort, of whom 69 had a follow-up of at least 1 year. Using the Kaplan-Meier method, OS was significantly longer in the prospective cohort than in the retrospective cohort (23 vs. 14 months, $p=0.012$ ). In addition, median PFS was significantly longer in the prospective cohort than in the retrospective cohort (17 vs. 11 months, $p=0.012$ ). Conclusion In the PERTAIN study, the preliminary results indicate that introducing FDG PET/CT-guided concurrent CRT for patients with stage III NSCLC in LMIC resulted in a significant improvement in OS and PFS. The final study results based on complete data are expected in 2020 .
\end{abstract}

Keywords Low- and middle-income countries · Non-small-cell lung cancer · PET/CT-guided chemoradiotherapy

This article is part of the Topical Collection on Oncology - Chest

Electronic supplementary material The online version of this article (https://doi.org/10.1007/s00259-019-04421-5) contains supplementary material, which is available to authorized users.

T. Konert

t.konert@nki.nl

G. G. Hanna

gerry.hanna@petermac.org

Extended author information available on the last page of the article

\section{Introduction}

Worldwide, lung cancer is the most commonly diagnosed cancer (2.1 million new cases in 2018) and the leading cause of cancer death (1.8 million deaths estimated in 2018) [1]. Five-year survival of lung cancer was $20-33 \%$ in countries such as Japan, Canada, USA, China, Korea, Israel, Sweden, Switzerland and Austria. However, most other countries had a 5 -year survival ranging between $10 \%$ and $20 \%$. Survival was 
less than $10 \%$ in countries such as Brazil, India and Thailand. Globally, lung cancer survival rates between 1995 and 1999 and between 2000 and 2014 indicate no improvement with time, but in high-income countries 5-year overall survival (OS) has increased by $5-10 \%$ in absolute terms over the same time period [2].

Currently, ${ }^{18} \mathrm{~F}$-fluorodeoxyglucose positron emission tomography/computed tomography (FDG PET/CT) is widely used for staging patients with non-small-cell lung cancer (NSCLC) and to a lesser extent for radiotherapy (RT) target volume delineation (TVD) [3, 4]. PET/CT scanners have also become available in several low- and middle-income countries (LMIC), although FDG PET/CT is mainly used for staging purposes rather than as a part of treatment planning in NSCLC [5]. The International Atomic Energy Agency (IAEA) convened an expert panel to appraise the clinical utility of FDG PET/CT for staging and RT planning (RTP) in patients with lung cancer. This coordinated research programme resulted in the design of the international PET/CT in RTP (PERTAIN) study (NCT02247713) to assess the feasibility of including FDG PET/CT in the RTP process in patients with stage III NSCLC in LMIC.

The current standard treatment for stage III NSCLC is concurrent chemoradiotherapy (CRT) [6]. In order to take advantage of the recent developments in RT techniques which have improved the accuracy of treatment delivery, it is essential to ensure TVD is as accurate as possible to avoid geographic miss of disease. Advanced RT techniques have improved local tumour control and have reduced treatment toxicity by enabling the delivery of higher radiation doses to the tumour while sparing adjacent normal tissue [7]. Examples include intensity-modulated RT (IMRT) [8], and image-guided RT, which improves the precision of treatment delivery and allows the use of smaller expansion margins [9].

TVD involves contouring the gross tumour volume (GTV), as specified in ICRU report 50 [10]. GTV delineation is sensitive to interobserver variability (IOV) [11, 12]. A significant reduction in IOV can be achieved with information from both PET and CT [12-15]. Automatic PET segmentation methods have also been proposed to reduce IOV [16], but always need verification by a radiation oncologist (RO) [17]. PET is specifically helpful in TVD when the tumour is not easily distinguished from surrounding healthy tissue on CT images, due to its higher soft tissue contrast [18]. Even with the use of PET imaging there is still IOV due to differences in the TVD method and to FDG uptake in normal structures adjacent to the tumour $[19,20]$. The use of a rigorous contouring protocol in which a multidisciplinary team including a $\mathrm{RO}$ and a nuclear medicine physician (NMP) follow a detailed set of instructions has been shown to help minimize IOV [21]. A recent IAEA publication has provided guidance on the use and role of FDG PET/CT imaging for RTP in NSCLC patients [17]. The impact of the use of the IAEA study protocol on
TVD accuracy and reproducibility has been evaluated in multiple centres in LMIC. Multiple training interventions on PET/ CT-based TVD in NSCLC improves delineation accuracy and reduces IOV [16]. Hence, we hypothesized that TVD following the IAEA study protocol would increase accuracy and reproducibility of TVD in the clinic, leading to improvement in local control.

There are many reports describing IOV within and outside the context of clinical trials, but few studies have investigated the impact of IOV on clinical outcome $[20,22,23]$ or methods that could minimize IOV by means of training [16, 24-26]. The clinical impact of such training remains unknown. Hence, we hypothesized that TVD following the IAEA study protocol would increase the accuracy and reproducibility of TVD and lead to improvement in local control and thus OS. We present the preliminary results of the PERTAIN study. The aim of this study was to assess the impact of introducing FDG PET/CTguided concurrent CRT, supported by training and quality control (QC), on the OS and PFS in patients with stage III NSCLC.

\section{Materials and methods}

\section{Ethical aspects}

The PERTAIN study was approved by the medical ethics committee of Netherlands Cancer Institute/Antoni van Leeuwenhoek Hospital (ref. M14PRI). In addition, each centre received ethical clearance from their local medical research ethics committee. Written informed consent was obtained from all patients included in the prospective phase.

\section{Study framework}

Nine medical centres in seven countries met the technical requirements to participate in the PERTAIN study, including six middle-income countries: Brazil, India, Jordan, Pakistan, Turkey and Vietnam. The seventh country, Estonia, is classified as a high-income country by the World Bank, but did not routinely use PET/CT for RTP. The first component of the study was data collection from a retrospective cohort, which included consecutive patients with stage III NSCLC who had been treated in the participating centres between January 2010 and July 2014 according to existing local protocols. Over a 1year period, nine pairs of trainees each including a RO and a NMP with limited experience in PET/CT-based TVD in NSCLC from seven different countries took part in multiple training interventions. Teams were given hands-on training in delineating the primary tumour according to IAEA protocol guidelines. An online webinar training session was held on TVD in NSCLC, and lectures for ROs and NMPs on current best practice in NSCLC were given [19]. All PET/CT scanners 
received annual European Association of Nuclear Medicine (EANM) Research Ltd. (EARL) FDG PET/CT accreditation. After the training intervention and scanner calibration, patients with stage III NSCLC were included in the prospective cohort between August 2015 and October 2018. The study entry criteria are summarized in the Supplementary material S1 (Form 1). Patients who did not meet the study entry criteria were excluded from the study.

\section{Case report forms}

Patient data were collected using electronic case report forms (eCRFs). Five different eCRFs were designed to collect information on patient eligibility, before and after treatment, and follow-up. More details on eCRFs and their format can be found in the Supplementary material S1.

\section{Clinical endpoints}

The primary endpoint was OS, defined as the time between the start of treatment and date of death or loss to follow-up. The secondary endpoint was progression-free survival (PFS). PFS was defined as the time from the start of treatment to local failure, time to regional failure, and/or time to distant failure. Local failure was defined as progression in the primary tumour, and regional failure as progression in involved lymph nodes as assessed on follow-up scans. Distant failure was defined according to the 8th edition of the TNM classification for NSCLC [27]. The intervals for the follow-up assessments and imaging were as per local follow-up guidelines.

\section{Chemotherapy and radiotherapy details}

Patients in the retrospective cohort were treated according to respective institutional practice with sequential concurrent CRT, neoadjuvant chemotherapy or RT alone, but with curative intent. In the prospective cohort, patients were treated with concurrent CRT to a total dose of at least $60 \mathrm{~Gy}$ in fractions of 2 Gy over 6 weeks. Centres were free to select chemotherapy regimens according to local practice.

\section{PET/CT image acquisition}

Patients underwent whole-body FDG PET/CT using one of the following scanners: Discovery ST, Discovery 710, Discovery STE (GE Medical Systems, Chicago, IL, USA), Biograph $40 \mathrm{mCT}$, and Biograph $64 \mathrm{mCT}$ (Siemens Medical Solutions, Erlangen, Germany). The reconstruction voxel size of the PET data varied from $2.0 \times 2.0 \times 3.3 \mathrm{~mm}$ to $5.5 \times 5.5 \times$ $3.3 \mathrm{~mm}$. Patients fasted for at least $8 \mathrm{~h}$ to ensure low levels of serum glucose. The total injected dose ranged between $226 \mathrm{MBq}$ and $441 \mathrm{MBq}$ (data not available for all patients). Patients were scanned approximately $60 \mathrm{~min}$ after injection of ${ }^{18}$ F-FDG according to EANM guidelines [28]. The acquisition times of the PET/CT scanners were in the range 2-5 min per bed position.

\section{Assessment of the retrospective and prospective cohort}

To assess the overall impact of the multiple training interventions and the routine use of FDG PET/CT-based concurrent CRT, survival outcomes in the retrospective cohort were compared with those in the prospective cohort. Although the training programme focused mainly on standardized PET/CTbased TVD, in general, the whole RTP procedure was also standardized to ensure the use of current treatment standards. Differences in the RTP procedures between the retrospective and prospective cohorts are summarized in Table 1.

\section{Quality control of target volume delineation}

To ensure that participating centres in the prospective study complied with the IAEA study protocol, central QC review of

Table 1 Differences in staging, radiotherapy planning, treatment and target volume delineation procedures between the retrospective and prospective cohorts

\begin{tabular}{lll}
\hline Comparison & Retrospective cohort & Prospective cohort \\
\hline Staging & With or without PET/CT & With PET/CT \\
RTP & With or without PET/CT & PET/CT in RTP-position \\
Time interval & Per local protocol, delays of $>1$ month possible & Within 4 weeks of last PET/CT \\
Delivered dose & Per local protocol & $\geq 60$ Gy \\
Treatment & RT, sequential CRT, CCRT & CCRT only \\
TVD & Per local protocol & Per IAEA study protocol (PET/CT-based) \\
PET/CT quality assurance & EARL accreditation not compulsory & EARL accreditation compulsory \\
Nodal irradiation & Both elective and involved nodal RT & Involved nodal RT
\end{tabular}

All procedures in the prospective cohort were standardized in all centres in accordance with the IAEA study guidelines [17]

(C)CRT concurrent) chemoradiotherapy, $R T$ radiotherapy, RTP radiotherapy planning, TVD tumour volume delineation 
TVD was performed for the first three patients included per centre, and thereafter as needed. In the QC process anonymized PET/CT data and RT structure sets were made available through a secure online storage service and were reviewed by at least two members of the study trial management group.

\section{Statistical analysis}

Any differences in continuous variables between the retrospective and prospective cohorts were assessed using the independent $t$ test. Any differences in categorical variables were assessed using the chi-squared test. Strong prognostic factors were identified using univariate Cox regression analysis. Kaplan-Meier analysis was performed to assess OS and PFS in the retrospective and prospective groups. The log-rank statistic was used to assess the significance of any differences. Statistical analysis was performed using IBM SPSS statistics for Windows, version 22.0 (IBM Corp., Armonk, NY). Values of $p$ less than 0.05 were considered significant.

\section{Results}

\section{Patient inclusion}

The retrospective cohort included 230 patients with stage III NSCLC treated with sequential or concurrent CRT or RT alone. The prospective cohort included 69 patients with stage III NSCLC. In all centres, a high percentage of patients (up to $51 \%$ ) were upstaged to stage IV after staging with PET/CT became the standard. Overall, five patients did not meet the study inclusion criteria, and were therefore excluded. Reasons for exclusion were inability to provide informed consent (one patient), unable to start treatment within 4 weeks of PET/CT (two patients), and an ECOG performance status (PS) of 2
Table 2 Patient and tumour characteristics

\begin{tabular}{|c|c|c|c|}
\hline & Retrospective cohort & Prospective cohort & $p$ value $^{\mathrm{a}}$ \\
\hline No. of patients & 230 & 69 & - \\
\hline Mean age (range) & $61(31-86)$ & $64(43-86)$ & 0.136 \\
\hline \multicolumn{4}{|l|}{ Gender } \\
\hline $\begin{array}{l}\text { Male } \\
\text { Female }\end{array}$ & $\begin{array}{c}191(83 \%) \\
39(17 \%)\end{array}$ & $\begin{array}{l}57(83 \%) \\
12(17 \%)\end{array}$ & 0.831 \\
\hline Smoker & $182(79 \%)$ & $67(97 \%)$ & $<0.001$ \\
\hline COPD & $75(33 \%)$ & $47(68 \%)$ & $<0.001$ \\
\hline \multicolumn{4}{|l|}{ ECOG performance status } \\
\hline $\begin{array}{l}0 \\
1\end{array}$ & $\begin{array}{c}72(31 \%) \\
158(69 \%)\end{array}$ & $\begin{array}{l}21(30 \%) \\
48(70 \%)\end{array}$ & 0.841 \\
\hline \multicolumn{4}{|l|}{ Disease stage } \\
\hline $\begin{array}{l}\text { IIIA } \\
\text { IIIB } \\
\text { IIIC }\end{array}$ & $\begin{array}{l}145(63 \%) \\
53(23 \%) \\
32(14 \%)\end{array}$ & $\begin{array}{l}29(42 \%) \\
27(39 \%) \\
13(19 \%)\end{array}$ & 0.021 \\
\hline \multicolumn{4}{|l|}{ T stage } \\
\hline $\begin{array}{l}1 \\
2 \\
3 \\
4\end{array}$ & $\begin{array}{c}4(2 \%) \\
43(19 \%) \\
87(38 \%) \\
96(42 \%)\end{array}$ & $\begin{array}{c}3(4 \%) \\
11(16 \%) \\
21(30 \%) \\
34(49 \%)\end{array}$ & 0.369 \\
\hline \multicolumn{4}{|l|}{$\mathrm{N}$ stage } \\
\hline $\begin{array}{l}0 \\
1 \\
2 \\
3\end{array}$ & $\begin{array}{c}16(7 \%) \\
39(17 \%) \\
135(59 \%) \\
40(17 \%)\end{array}$ & $\begin{array}{c}4(6 \%) \\
4(6 \%) \\
42(61 \%) \\
19(27 \%)\end{array}$ & 0.082 \\
\hline \multicolumn{4}{|l|}{ Histology } \\
\hline $\begin{array}{l}\text { Squamous cell carcinoma } \\
\text { Adenocarcinoma } \\
\text { Large cell carcinoma } \\
\text { Not otherwise specified }\end{array}$ & $\begin{array}{c}90(39 \%) \\
97(42 \%) \\
15(7 \%) \\
28(12 \%)\end{array}$ & $\begin{array}{c}32(46 \%) \\
35(51 \%) \\
0(0 \%) \\
2(3 \%)\end{array}$ & 0.013 \\
\hline
\end{tabular}

COPD chronic obstructive pulmonary disease, ECOG Eastern Cooperative Oncology Group

${ }^{a}$ Calculated using the independent $t$ test for continuous variables or the chi-squared test for categorical variables 
(two patients). An overview of the patient and tumour characteristics is given in Table 2.

\section{Quality control of target volume delineation}

All participating centres completed the first step of the QC procedure, in which the first three patients were accepted in the PERTAIN study. In total, 35 patients were submitted for TVD QC. Nine patients (26\%) were excluded after evaluation. The reasons for not accepting the TVD as acceptable were: incorrect staging (two patients), involved lymph nodes not included (two patients), tumours too large to treat radically ( $\geq 60 \mathrm{~Gy}$ ) without exceeding dose constraints (three patients), and noncompliance with IAEA study guidelines (two patients). All other patients in the prospective cohort were accepted for inclusion and treatment with concurrent CRT.

\section{Treatment parameters}

In the retrospective cohort, 18 patients were treated with RT only (8\%), 65 patients received sequential CRT (28\%), and 147 patients (64\%) received concurrent CRT. In contrast, all patients in the prospective cohort received concurrent CRT with curative intent. In both the retrospective and prospective cohorts various chemotherapy regimens were intravenously administered weekly: either carboplatin-based or cisplatinbased in combination with paclitaxel, etoposide, docetaxel, pemetrexed or gemcitabine. Of the 230 patients in the retrospective cohort, 32 (14\%) were treated using an IMRT technique and 198 (86\%) using three-dimensional conformal RT (3DCT). By comparison, of the 69 patients in the prospective cohort, 29 (42\%) were treated with IMRT, 2 (3\%) with volumetric modulated arc therapy (VMAT), and 38 (55\%) with

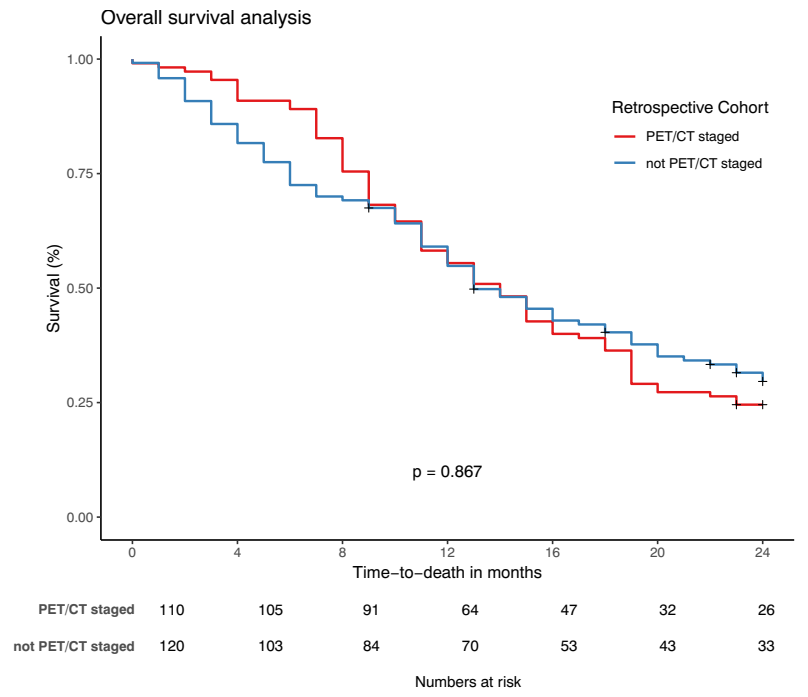

3DCT. The prescribed dose fractionation scheme varied between 50 Gy in 30 fractions and 70 Gy in 35 fractions in the retrospective cohort, with a mean prescribed dose of $61.4 \pm$ $2.8 \mathrm{~Gy}$. In the prospective cohort the dose fractionation scheme varied between $60 \mathrm{~Gy}$ in 30 fractions and $66 \mathrm{~Gy}$ in 33 fractions, with a mean prescribed dose of $60.7 \pm 1.7 \mathrm{~Gy}$.

\section{Impact on survival}

Prognostic factors were evaluated in the retrospective and prospective cohorts separately. In the retrospective cohort, age and ECOG PS were significant prognostic factors $(p=$ 0.039 and 0.024 , respectively), and T stage demonstrated borderline significance $(p=0.053)$. In the prospective cohort, univariate Cox regression analysis showed no significant prognostic factors. No significant differences between the retrospective and the prospective cohorts in any of these prognostic variables were found, and therefore these variables were considered balanced. However, TNM staging was significantly higher in the prospective cohort than in the retrospective cohort $(p=0.021)$, and histological subtype was significantly different between the cohorts $(p=0.013)$ The difference in histological subtype was due to the absence of large-cell and lack of not otherwise specified types in the prospective cohort (see Table 2).

In the retrospective cohort, Kaplan-Meier analysis showed no significant differences in OS or PFS between patients who were and were not PET/CT-staged ( $p=0.867$ and 0.304 , respectively; Fig. 1). Only $18.1 \%$ of the retrospective data were censored; in contrast, $52.2 \%$ of the prospective data were censored. Median survival was 14 months (95\% CI 12-15 months) in the retrospective cohort and 23 months (95\% CI 15-30 months) in the

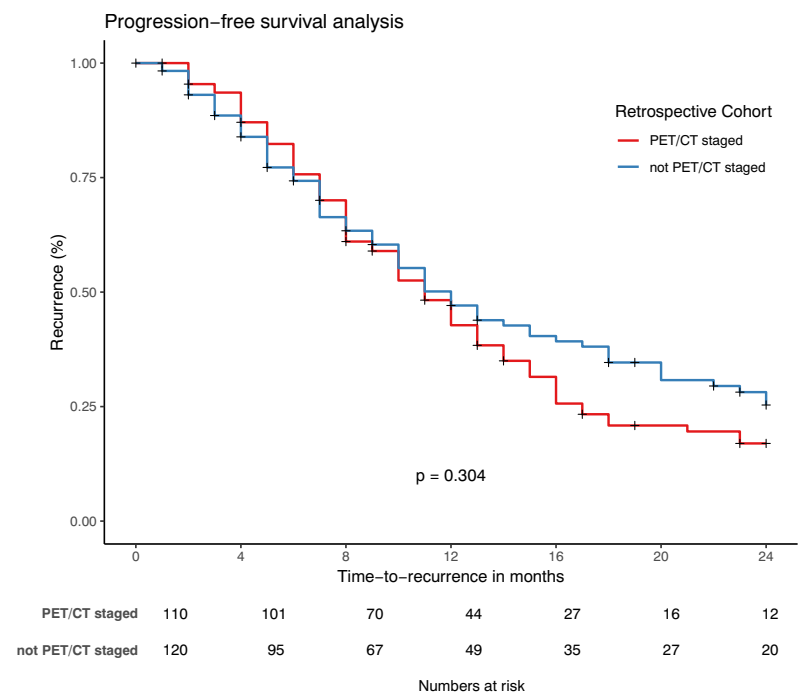

Fig. 1 Kaplan-Meier analysis of the difference in overall survival (left $)$ and progression-free survival (right) in the retrospective cohort between patients who were and were not PET/CT-staged. No significant differences were observed in overall survival $(p=0.867)$ or progression-free survival $(p=0.304)$ 
prospective cohort ( $p=0.012, \log$-rank test). Two-year OS was $27 \%$ in the retrospective cohort and and $47 \%$ in the prospective cohort. The corresponding Kaplan-Meier analysis is shown in Fig. 2.

Kaplan-Meier analysis of PFS in the retrospective and the prospective cohorts is shown in Fig. 3. Median time to progression was 11 months (95\% CI 9-12 months) in the retrospective cohort and 17 months (95\% CI 10-23 months) in the prospective cohort ( $p=0.012$, log-rank test). Two-year PFS was $22 \%$ in the retrospective cohort and $45 \%$ in the prospective cohort.

\section{Discussion}

This study investigated the impact of introducing FDG PET/ CT-guided concurrent CRT, supported by training and QC, on the OS in patients with stage III NSCLC. Preliminary results demonstrated a positive trend in a cohort comparison towards improved OS and PFS in the prospective cohort, suggesting a benefit from implementing FDG PET/CT-guided concurrent CRT in patients with stage III NSCLC in centres in LMIC with limited experience with PET/CT. TVD QC showed that IAEA study guidelines were implemented successfully in the clinic in $74 \%$ of patients. This demonstrates compliance with the study guidelines in the clinic, but also emphasizes the importance of QC in multi-centre trials to ensure compliance with the study protocol. Using TVD QC we were therefore able to confirm compliance with IAEA study guidelines in the clinic. This procedure led to the removal of five patients ineligible for curative CCRT who could otherwise have influenced the outcome of this study. Four issues were observed in TVD QC: incorrect staging, involved lymph nodes not included,

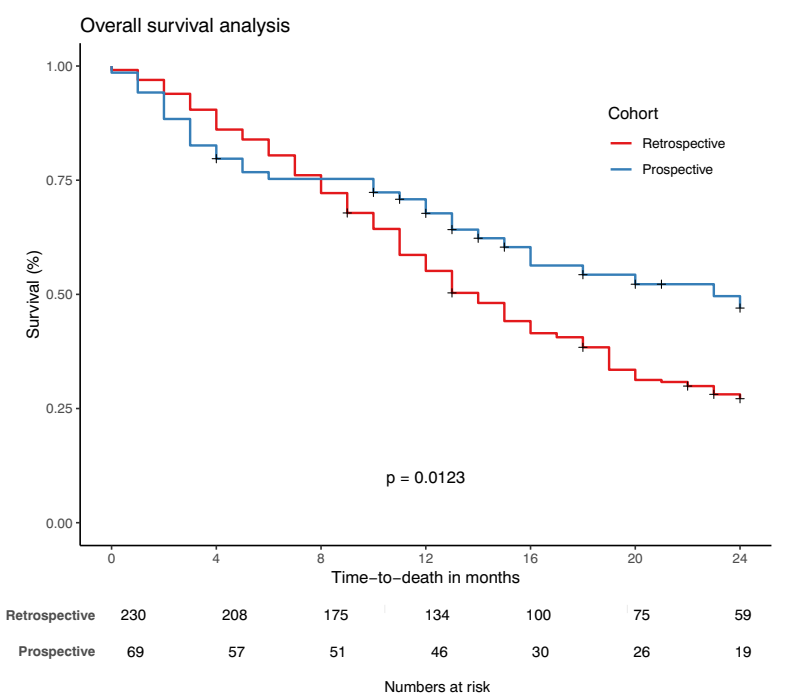

Fig. 2 Kaplan-Meier analysis of the difference in overall survival between the retrospective and the prospective cohorts. A survival benefit was observed in the prospective patient cohort $(p=0.012)$

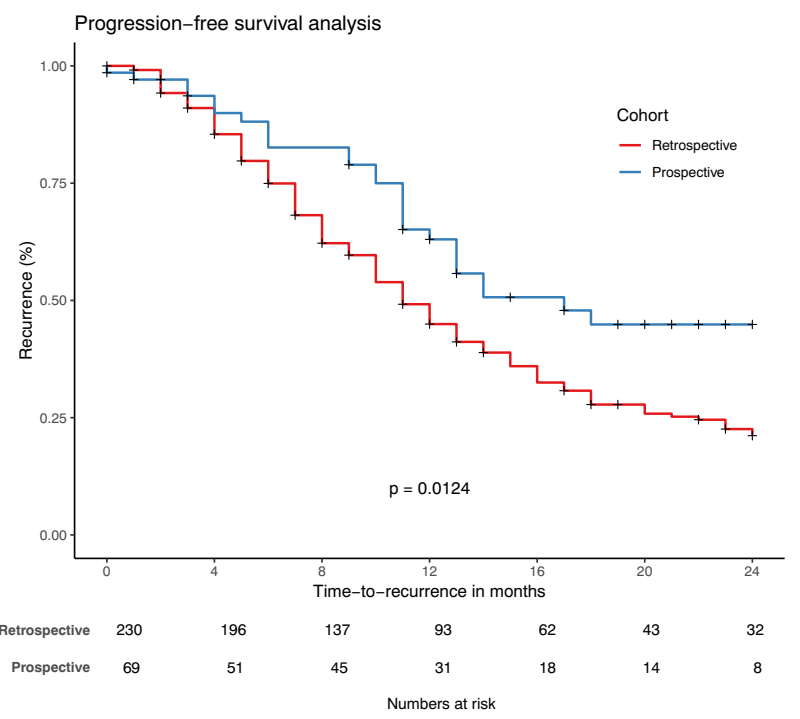

Fig. 3 Kaplan-Meier analysis of the difference in progression-free survival between the retrospective and the prospective cohort. A survival benefit was observed in the prospective patient cohort $(p=0.012)$

tumours too large to treat radically without exceeding dose constraints, and tumours not delineated following the reGTV approach. The QC reviews continued to inform study participants during patient accrual and served as educational material when these issues occurred, which emphasizes once more the importance of QC during clinical studies.

Significant differences in TNM stage were observed between the retrospective and prospective cohorts. The retrospective cohort included predominantly stage IIIA patients, whereas the prospective cohort had a more balanced distribution of patients with stages IIIA, B and C. Despite a survival benefit of stage IIIA over IIIB and IIIC, results demonstrated better survival in the prospective cohort. Nevertheless, $48 \%$ of the patients in the retrospective cohort were not PET/CTstaged, and could have been incorrectly staged. Indeed, in all centres, a high percentage (up to $51 \%$ ) of patients were upstaged to stage IV after staging with PET/CT became the standard.

The improved OS and PFS were possibly due to several factors. Besides the introduction of PET/CT for TVD and improved patient selection with FDG PET/CT, patients in the prospective cohort all received CCRT (64\% in the retrospective cohort; $100 \%$ in the prospective cohort) and were also treated with more advanced RT techniques such as IMRT and VMAT (14\% in the retrospective cohort; $45 \%$ in the prospective cohort), which could also have led to survival benefits [6]. Even so, there was no significant difference in the prescribed doses between the cohorts, and evidence is lacking on the survival benefit of IMRT/VMAT versus 3DCT in lung cancer patients [29]. On the other hand, more confidence was gained in PET/CT-based contouring, increasing delineation accuracy which hopefully resulted in reduced geographic miss of 
tumour. This may explain the improved local control seen in Fig. 2. In addition, the PERTAIN trial improved or reaffirmed collaborative working relationships between nuclear medicine and radiation oncology departments in the participating centres. This collaboration may not only have led to improved delineation, but also may have improved patient management by streamlining the patient pathway from diagnosis to treatment. Evaluation of the impact of this collaboration on outcome was beyond the scope of this study, and further research is required to obtain definitive evidence [30, 31].

In the PERTAIN trial there was a heterogeneous group of participating centres with different levels of experience in PET/CT scan acquisition. Furthermore, the training interventions were limited to RO and NMP chief scientific investigators. A train-the-trainers approach was used to disseminate the knowledge further in the departments involved in the PERTAIN study. The improvement in survival outcomes shown in this analysis suggests that this training approach had a clinically meaningful impact in the participating centres. We suggest that it is feasible to disseminate education regarding new radiation oncology techniques using the multiple intervention method we used [17].

One potential confounding impact in the comparison of outcomes between the cohorts may have been the impact of $\mathrm{PET} / \mathrm{CT}$ staging alone. It is interesting to note that in the retrospective cohort, no significant difference in OS was observed between patients who were and were not PET/CTstaged (Fig. 1), and hence this confounding effect may have been negligible in this cohort, but it is acknowledged that because of the size of these groups, the study may not have been powered to detect a true difference. Another potential confounding factor was the selection bias that may have been present between the retrospective cohort and prospective cohort with regard to patients who died during treatment. This may explain the worse survival seen in the first months in the prospective cohort. Five patients died during RT in the prospective cohort after being included in the analysis, and patients who died during treatment were not selected for the retrospective cohort. However, this would have had the effect of reducing the apparent survival difference between the two arms of the study. Another influence that may have contributed to worse survival in the first months may have been the higher incidence of smokers and patients with COPD in the prospective cohort.

\section{Conclusion}

The initial analysis of the PERTAIN study showed that a combined package of FDG PET/CT-planned RT, the routine use of concurrent CRT with training support, and a robust QC process led to improved OS and PFS in patients with stage III NSCLC patients in low- and middle-income countries.
Acknowledgments The authors acknowledge the following for providing technical assistance in this study: Elba Etchebehere, Ana Brito, Nauman Amjad, Camila Mosci, Valdelania Lamounier, Flavia Gabriella, Diyaa Juaidi, Olga Morozova, Yaroslav Pynda, Nguyen Tan Chau and Terez Sera. The authors are grateful to Vincent van der Noort for providing statistical support. The authors also acknowledge Arturo Chiti, Ursula Nestle and Vincent Grégoire for contributing to the design of the PERTAIN study and for providing expert opinion on PERTAIN study guidelines.

Funding The International Atomic Energy Agency, Vienna, Austria, funded all meetings of national country coordinators, data collection and processing for this project.

\section{Compliance with ethical standards}

Conflict of interest None.

Ethical approval Ethical approval for the study was obtained from the Medical Ethics Committee of the Netherlands Cancer Institute (M14PRI).

Informed consent Informed consent was obtained from all individual participants included in the study.

Open Access This article is distributed under the terms of the Creative Commons Attribution 4.0 International License (http:// creativecommons.org/licenses/by/4.0/), which permits unrestricted use, distribution, and reproduction in any medium, provided you give appropriate credit to the original author(s) and the source, provide a link to the Creative Commons license, and indicate if changes were made.

\section{References}

1. Bray F, Ferlay J, Soerjomataram I, Siegel RL, Torre LA, Jemal A. Global cancer statistics 2018: GLOBOCAN estimates of incidence and mortality worldwide for 36 cancers in 185 countries. CA Cancer J Clin. 2018;68(6):394-424.

2. Allemani C, Matsuda T, Di Carlo V, Harewood R, Matz M, Nikšić $\mathrm{M}$, et al. Global surveillance of trends in cancer survival 2000-14 (CONCORD-3): analysis of individual records for $37 \quad 513 \quad 025$ patients diagnosed with one of 18 cancers from 322 populationbased registries in 71 countries. Lancet. 2018;391(10125):1023-75.

3. Ung YC, Bezjak A, Coakley N, Evans WK; the Lung Cancer Disease Site Group of Cancer Care Ontario. Positron emission tomography with 18 fluorodeoxyglucose in radiation treatment planning for non-small cell lung cancer: a systematic review. J Thorac Oncol. 2011;6:86-97.

4. Gregory DL, Hicks RJ, Hogg A, Binns DS, Shum PL, Milner A, et al. Effect of PET/CT on management of patients with non-small cell lung cancer: results of a prospective study with 5-year survival data. J Nucl Med. 2012;53:1007-15.

5. Badar F, Mahmood S, Yusuf MA, Sultan F. Epidemiology of cancers in Lahore, Pakistan, 2010-2012: a cross-sectional study. BMJ Open. 2016;6:e11828.

6. Aupérin A, Le Péchoux C, Rolland E, Curran WJ, Furuse K, Fournel P, et al. Meta-analysis of concomitant versus sequential radiochemotherapy in locally advanced non-small cell lung cancer. J Clin Oncol. 2010;28:2181-90.

7. Liao ZX, Komaki RR, Thames HD Jr, Liu HH, Tucker SL, Mohan $\mathrm{R}$, et al. Influence of technologic advances on outcomes in patients with unresectable, locally advanced non-small-cell lung cancer 
receiving concomitant chemoradiotherapy. Int J Radiat Oncol Biol Phys. 2010;76(3):775-81.

8. Chun SG, Hu C, Choy H, Komaki RU, Timmerman RD, Schild SE, et al. Comparison of 3-D conformal and intensity modulated radiation therapy outcomes for locally advanced non-small cell lung cancer in NRG oncology/RTOG 0617. Int J Radiat Oncol Biol Phys. 2015;93(3 Suppl):S1-2.

9. Ahmad SS, Duke S, Jena R, Williams MV, Burnet NG. Advances in radiotherapy. BMJ. 2012;345:e7765.

10. International Commission on Radiation Units and Measurements. ICRU Report No. 50. Prescribing, Recording and Reporting Photon Beam Therapy. Washington, DC: International Commission on Radiation Units and Measurements; 1993.

11. Steenbakkers RJ, Duppen JC, Fitton I, Deurloo KE, Zijp L, Uitterhoeve AL, et al. Observer variation in target volume delineation of lung cancer related to radiation oncologist-computer interaction: a 'big brother' evaluation. Radiother Oncol. 2005;77:18290.

12. Rasch C, Belderbos J, van Giersbergen A, De Kok I, Laura T, Boer $\mathrm{M}$, et al. The influence of a multi-disciplinary meeting for quality assurance on target delineation in radiotherapy treatment preparation. Int J Radiat Oncol. 2009(3 Suppl);75:S452-3.

13. Hanna G, McAleese J, Carson KJ, Stewart DP, Cosgrove VP, Eakin RL, et al. (18)F-FDG PET-CT simulation for non-small-cell lung cancer: effect in patients already staged by PET-CT. Int J Radiat Oncol Biol Phys. 2010;77:24-30.

14. Greco C, Rosenzweig K, Cascini GL, Tamburrini O. Current status of PET/CT for tumor volume definition in radiotherapy treatment planning for non-small cell lung cancer (NSCLC). Lung Cancer. 2007;57:125-34.

15. Fox JL, Rengan R, O’Meara W, Yorke E, Erdi Y, Nehmeh S, et al. Does registration of PET and planning CT images decrease interobserver and intraobserver variation in delineating tumor volumes for non-small-cell lung cancer? Int J Radiat Oncol Biol Phys. $2005 ; 62: 70-5$.

16. Hatt M, Lee JA, Schmidtlein CR, Naqa IE, Caldwell C, De Bernardi E, et al. Classification and evaluation strategies of autosegmentation approaches for PET: report of AAPM task group no. 211. Med Phys. 2017;44(6):e1-e42.

17. Konert T, Vogel W, MacManus MP, Nestle U, Belderbos J, Grégoire V, et al. PET/CT imaging for target volume delineation in curative intent radiotherapy of non-small cell lung cancer: IAEA consensus report 2014. Radiother Oncol. 2015;116:27-34.

18. Caldwell CB, Mah K, Ung YC, Danjoux CE, Balogh JM, Ganguli $\mathrm{SN}$, et al. Observer variation in contouring gross tumor volume in patients with poorly defined non small-cell lung tumors on CT: the impact of 18FDG-hybrid PET fusion. Int J Radiat Oncol Biol Phys. 2001;51:923-31.

19. Konert T, Vogel WV, Everitt S, MacManus MP, Thorwarth D, Fidarova E, et al. Multiple training interventions significantly improve reproducibility of PET/CT-based lung cancer radiotherapy target volume delineation using an IAEA study protocol. Radiother Oncol. 2016;121:39-45.
20. Van de Steene J, Linthout N, de Mey J, Vinh-Hung V, Claassens C, Noppen $\mathrm{M}$, et al. Definition of gross tumor volume in lung cancer: inter-observer variability. Radiother Oncol. 2002;62(1):37-49.

21. Bayne M, Hicks RJ, Everitt S, Fimmell N, Ball D, Reynolds J, et al. Reproducibility of "intelligent" contouring of gross tumor volume in non-small-cell lung cancer on PET/CT images using a standardized visual method. Int J Radiat Oncol Biol Phys. 2010;77:1151-7.

22. Jameson MG, Kumar S, Vinod SK, Metcalfe PE3, Holloway LC. Correlation of contouring variation with modeled outcome for conformal non-small cell lung cancer radiotherapy. Radiother Oncol. 2014;112:332-6.

23. Peters LJ, O'Sullivan B, Giralt J, Fitzgerald TJ, Trotti A, Bernier J, et al. Critical impact of radiotherapy protocol compliance and quality in the treatment of advanced head and neck cancer: results from TROG 02.02. J Clin Oncol. 2010;28(18):2996-3001.

24. Schimek-Jasch T, Troost EG, Rücker G, Prokic V, Avlar M, Duncker-Rohr V, et al. A teaching intervention in a contouring dummy run improved target volume delineation in locally advanced non-small cell lung cancer: reducing the interobserver variability in multicentre clinical studies. Strahlenther Onkol. 2015;191:525-33.

25. Spoelstra FO, Senan S, Le Péchoux C, Ishikura S, Casas F, Ball D, et al. Variations in target volume definition for postoperative radiotherapy in stage III non-small cell lung cancer: analysis of an international contouring study. Int J Radiat Oncol Biol Phys. 2010;76: 1106-13.

26. Nestle U, Rischke HC, Eschmann SM, Holl G, Tosch M, Miederer $\mathrm{M}$, et al. Improved inter-observer agreement of an expert review panel in an oncology treatment trial - insights from a structured interventional process. Eur J Cancer. 2015;51:2525-33.

27. Detterbeck FC, Boffa JB, Kim AW, Tanoue LT. The Eighth Edition Lung Cancer Stage Classification. Chest. 2017;151(1):193-203.

28. Boellaard R, Delgado-Bolton R, Oyen WJ, Giammarile F, Tatsch $\mathrm{K}$, Eschner W, et al. FDG PET/CT: EANM procedure guidelines for tumor imaging: version 2.0. Eur J Nucl Med Mol Imaging. 2015;42(2):328-54

29. Ball D, Mac Manus M, Siva S, Plumridge N, Bressel M, Kron T. Routine use of intensity-modulated radiotherapy for locally advanced non-small-cell lung cancer is neither choosing wisely nor personalized medicine. J Clin Oncol. 2017;35(13):1492-3.

30. Prabhu Das I, Baker M, Altice C, Castro KM, Brandys B, Mitchell SA. Outcomes of multidisciplinary treatment planning in US cancer care settings. Cancer. 2018;124(18):3656-67.

31. Pillay B, Wootten AC, Crowe H, Corcoran N, Tran B, Bowden P, et al. The impact of multidisciplinary team meetings on patient assessment, management and outcomes in oncology settings: a systematic review of the literature. Cancer Treat Rev. 2016;42:56-72.

Publisher's note Springer Nature remains neutral with regard to jurisdictional claims in published maps and institutional affiliations.

\section{Affiliations}

T. Konert ${ }^{1}$ (D) W. V. Vogel ${ }^{1,2} \cdot$ D. Paez ${ }^{3} \cdot$ A. Polo $^{3} \cdot$ E. Fidarova $^{3} \cdot$ H. Carvalho ${ }^{4} \cdot$ P. S. Duarte ${ }^{4}$ A. C. Zuliani ${ }^{5}$. A. O. Santos ${ }^{5}$. D. Altuhhova ${ }^{6}$. L. Karusoo ${ }^{6} \cdot$ R. Kapoor ${ }^{7}$ - A. Sood ${ }^{7}$. J. Khader ${ }^{8} \cdot$ A. Al-lbraheem ${ }^{8} \cdot$ Y. Numair ${ }^{9}$. S. Abubaker ${ }^{9}$. C. Soydal ${ }^{10}$ - T. Kütük ${ }^{10}$ - T. A. Le ${ }^{11}$ - N. X. Canh ${ }^{11}$ • B. Q. Bieu ${ }^{12}$ • L. N. Ha ${ }^{12}$ • J. S. A. Belderbos ${ }^{2}$. M. P. MacManus ${ }^{13,14}$ • D. Thorwarth ${ }^{15}$. G. G. Hanna ${ }^{13,14}$ 
1 Nuclear Medicine Department, Netherlands Cancer Institute, Plesmanlaan 121, 1066 CX Amsterdam, The Netherlands

2 Department of Radiation Oncology, Netherlands Cancer Institute, Amsterdam, The Netherlands

3 Division of Human Health, Department of Nuclear Sciences and Applications, International Atomic Energy Agency, Vienna, Austria

4 Department of Radiology and Oncology, Faculty of Medicine, University of São Paulo - Institute of Cancer of Sao Paulo State, São Paulo, Brazil

5 Department of Radiation Oncology and Nuclear Medicine Department, Hospital das Clínicas, Campinas University, Campinas, Brazil

6 Department of Radiation Oncology and Radiology Department, North Estonia Medical Center, Tallinn, Estonia

7 Department of Radiation Oncology and Nuclear Medicine Department, Postgraduate Institute of Medical Education and Research, Chandigarh, India

8 Department of Radiation Oncology and Nuclear Medicine Department, King Hussein Cancer Center, Amman, Jordan
9 Department of Radiation Oncology and Nuclear Medicine Department, Institute of Nuclear Medicine and Oncology, Lahore, Pakistan

10 Department of Radiation Oncology and Nuclear Medicine Department, Ankara University School of Medicine, Mamak/ Ankara, Turkey

11 Department of Radiation Oncology and Nuclear Medicine Department, Cho Ray Hospital, University of Ho Chi Minh City, Ho Chi Minh City, Vietnam

12 Department of Radiation Oncology and Radiosurgery, Tran Hung Dao Hospital, Hanoi, Vietnam

13 Department of Radiation Oncology, Peter MacCallum Cancer Centre, 305 Grattan Street, Melbourne, VIC 3000, Australia

14 Sir Peter MacCallum Department of Oncology, University of Melbourne, Parkville, Australia

15 Section for Biomedical Physics, Department of Radiation Oncology, University Hospital Tübingen, Tübingen, Germany 